\title{
Comparative Efficacy of Trichogramma chilonis Bred on Corcyra cephalonica and Leucinodes orbonalis (Guenee) Eggs in controlling Shoot and Fruit Borer in Brinjal
}

\author{
Mou Biswas, Ipsita Mishra and Subhalaxmi Roy*
}

Department of Entomology, OUAT, Bhubaneswar, Odisha, India

*Corresponding author

\section{Keywords}

Trichogramma chilonis, Corcyra cephalonica and Leucinodes orbonalis

Article Info

Accepted:

15 January 2021

Available Online:

10 February 2021

\section{A B S T R A C T}

Laboratory and field experiments were conducted during 2015-17 to study the Comparative efficacy of Trichogramma chilonis bred on Corcyra cephalonica and Leucinodes orbonalis (Guennee) eggs in controlling shoot and fruit borer in brinjal in coastal Odisha. Trichogramma chilonis was reared in the eggs of Corcyra cephalonica and Leucinodes orbonalis for 30 generations and the parasitoid was evaluated in field conditions against Brinjal shoot and fruit borer. It was observed that the parasitoid, when reared on eggs of L.orbonalis has shorter life cycle but better efficiency in controlling the pest.

\section{Introduction}

Vegetables are cheap sources of vitamins and minerals. They not only help in maintaining good health but also protect the body systems against degenerative diseases. In a developing country like India, where pressure of population on land is continuously increasing, vegetables will play significant role in supplying balanced diet to the general public. As such the scope for expansion of vegetable industry in India is quite promising and therefore cultivation of brinjal as a cheap source of vegetable has a bright future. As far as vegetable production in the world is concerned, India is the second, next only to China. Brinjal (Solanum melongena L.) is a versatile and economically important vegetable among small-scale farmers and low income consumers of the entire universe (FAO, 2000). It is the leading vegetable in the country and ranks first among summer and winter vegetables in terms of total average. Asia has the largest brinjal production which comprises about $90 \%$ of the total production area and $87 \%$ of the world production (FAO, 2000). Among the states, West Bengal, Odisha, Bihar, Gujarat and Maharashtra are considered as major brinjal growing states in India. In Orissa, the crop is grown over an 
area of about 127.7 thousand hectares and its production is being recorded as 1852.2 thousand metric tonnes. However, the productivity of this most common vegetable is about $14.5 \mathrm{t} / \mathrm{ha}$ which is comparatively less than that reported in states like West Bengal (18.2 t/ha), Bihar (20.0 t/ha) and Gujarat (15.2 t/ha) (Anonymous, 2005).

Brinjal, an economically important commercial crop, is reported variedly to be infested by more than 36 pests (Regupathy et al., 1997) from the time of its planting to harvest. Among them, brinjal shoot and fruit borer (BSFB), Leucinodes orbonalis Guenee is considered to be the most serious pest of brinjal and it has become a very serious production constraint in all brinjal growing countries (Alam et al., 2003). It inflicts damage to both shoots and fruits (Srinivasan, 2008). The fruit infestation by this pest may be as high as 67\% (BARI 1991). It is very difficult to control since it feeds inside the shoots and fruits (Ghosh and Senapati, 2009). The crop is attacked by a large number of Insect-pests (Payal Devi et al., 2015), among them shoot and fruit borer Leucinodes orbonalis Guenee (Lepidoptera: Pyralidae) is the key pest throughout Asia (RP Mainali, 2014). In recent years increased use of chemical pesticides by the farmers for combating $L$. orbonalis has made the cultivation of eggplant more costlier and hazardous to farmers as well as consumers in Orissa, majority of the farmers (41.7\%) followed a seven to eight spraying schedule to control this borer species and about 56.7\% farmers have found it expensive to control $L$. orbonalis through the use of insecticides (George et al.,2002). But sole reliance on chemicals has resulted 'ecocatastrophe' leading to development of resistance in L.orbonalis to different insecticides (Tomlin, 2000), pest resurgence (Mishra, 2008), harmful pesticide residue (Prabhat et al., 2000), destruction of beneficial insects and environmental hazards. Moreover Eggplant is mostly grown on small land holdings and sale of produce harvested almost daily over a 5-6 month period provide the farmers with valuable cash income. Therefore, there is a need to develop appropriate pest management strategies for low input based resource poor farming systems where the crop is grown under rain fed conditions as well as for high input based irrigated crop conditions where the farmers take 2 to 3 crops a years. So management strategies should aim at use of ecologically sound, economically viable and socially acceptable safer alternatives.

\section{Materials and Methods}

The investigations were conducted in the Biological laboratory of Entomology Department, College of Agriculture, O.U.A.T., Bhubaneswar and the department experimental farm named Central Farm, located in front of the Agriculture College. The location being is east and south eastern coastal plain agro-climatic Zone, experiences a typical hot and most sub humid climate.

\section{Laboratory rearing of $L$. orbonalis}

Biology of $L$. orbonails was studied under laboratory conditions on fruits of Utkal Anushree variety. The laboratory culture was maintained from the field-collected caterpillars were reared in the laboratory to ascertain the relative size of different instars, based upon which the larval stages collected subsequently through periodical sampling, were categorized as larval instar 1 to 5 . All the larvae were reared till adult emergence, on sliced potato tubers as suggested by Rajalaxmi et al.,(2004). The larvae of different instars as collected from time to time were transferred with the help of fine camel hairbrush on a place of sliced potato tubers individually in plastic specimen trays. Fresh sliced piece of potato was provided to the larvae on every 
alternate day. Prior to the rearing of $L$. orbonalis larvae, the acceptance of potato tubers as food by the insect was examined by releasing a few larvae on sliced end of a well washed potato tubers. Infested fruits collected from the experimental field, are kept in different nylon net covered cages(1'x1'x10") each, kept under natural environmental condition in the area of Entomology Department, College of Agriculture, OUAT, Bhubaneswar. Net is used in the cage to allow proper ventilation of air and to avoid rotting of fruits. Each box carries 5- $7 \mathrm{Kg}$ of brinjal fruit below which paper pieces are kept for proper pupation of the matured larvae in the fruits. The matured fifth instar larvae after coming out of the brinjal fruit and twigs moves down and pupate under the paper pieces below. The entire process is monitored on regular basis, the brinjal fruits were changed at periodic intervals, and rotted fruits were removed to avoid fungal growth. Pupae were collected regularly and kept in another cage under laboratory condition. The freshly emerged adult moths from pupae in the cage were collected manually by test tubes and known numbers of male and female moths were transferred to glass jar $(20 \mathrm{~cm} \mathrm{x} 15 \mathrm{~cm})$ in pairs, covered with black colour muslin cloth, for mating and oviposition. The adults were fed with a cotton swab soaked in $10 \%$ honey solution provided as source of food for the adult moths during oviposition. The muslin cloth was examined daily for egg laying and the eggs were parasitized by Trichogamma chilonis card in polycovers under controlled temperature and humidity in the laboratory. The entire process of fresh collection of pupae to adult moth to oviposition in fresh glass jars covered by black new muslin cloth then collection of eggs and parasitisation by Trichogamma card were repeated from time to time on different dates and the data was recorded to study the extent of parasitisation by Trichogamma chilonis in subsequent generations to conclude as one of the effective biocontrol agent against brinjal shoot and fruit borer. The parasitoid of T.chilonis reared on C.cephalonica and L.orbonalis were evaluated in field trials during 2016 and 2017 aginst L.orbonalis. Brinjal variety Utkal Anushree was grown in plots of 0.25 acre and plots were taken 40 meters apart for prevention of parasitoids migrating between plots. There were eight treatments as follows:

$\mathrm{T}_{1}$-Trichogramma@50,000 / ha on Corcyra cephalonica,

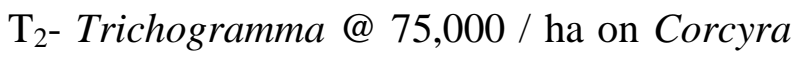
cephalonica,

$\mathrm{T}_{3^{-}}$Trichogramma @ 1,00,000 / ha on Corcyra cephalonica,

$\mathrm{T}_{4}$-Trichogramma @ 50,000 / ha on Leucinodes orbonalis,

$\mathrm{T}_{5}$-Trichogramma @ 75,000 / ha on Leucinodes orbonalis,

$\mathrm{T}_{6}$-Trichogramma @ $1,00,000$ / ha on Leucinodes orbonalis,

$\mathrm{T}_{7} \quad$ - Treated with Insecticide Flubendiamide+Rynaxypyr

$\mathrm{T}_{8^{-}}$Control

After application of the treatments $4 \mathrm{~m}^{2}$ area in each plot was selected as replication and ten such replications were taken in each plot for taking observations as shoot and fruit borer incidence.

\section{Results and Discussion}

Shoot infestation before treatment varied from $14.21 \%$ to $20.18 \%$ in different treatments. Fifteen days after treatment (DAT) the infestation was lowest $(9.61 \%)$ in $\mathrm{T}_{7}$ (Insecticidal treatment) followed by 
$\mathrm{T}_{6}(9.62 \%), \quad \mathrm{T}_{3}(12.01), \quad \mathrm{T}_{5}(12.02 \%)$, $\mathrm{T}_{4}(12.27 \%), \quad \mathrm{T}_{2}(13.88 \%) \quad$ and $\mathrm{T}_{1}(14.56 \%)$ respectively as against $20.66 \%$ in untreated control. Similarly at 30DAT shoot infestation was lowest in $\mathrm{T}_{7}(7.52 \%)$ followed by $\mathrm{T}_{6}(7.53 \%), \quad \mathrm{T}_{3}(10.81 \%), \quad \mathrm{T}_{5}(11.49 \%)$, $\mathrm{T}_{4}(11.52 \%), \quad \mathrm{T}_{2}(12.17 \%)$ and $\mathrm{T}_{1}(12.31 \%)$ respectively as against $19.13 \%$ in untreated control. Similar trend was recorded at 45 DAT, 60DAT, 75DAT and 90DAT. At 45DAT percent of shoot infestation ranges from lowest at $\mathrm{T}_{7}(5.34 \%)$ followed by $\mathrm{T}_{6}(5.36 \%), \quad \mathrm{T}_{3}(10.03 \%), \quad \mathrm{T}_{4}(10.30 \%)$, $\mathrm{T}_{5}(10.81 \%), \quad \mathrm{T}_{2}(11.85 \%), \quad \mathrm{T}_{1}(12.05 \%), \quad$ at 60DAT lowest shoot infestation was recorded at $\mathrm{T}_{7}(4.64 \%)$ followed by $\mathrm{T}_{6}(4.65 \%)$, $\mathrm{T}_{4}(9.04 \%), \quad \mathrm{T}_{5}(9.11 \%), \quad \mathrm{T}_{3}(9.24 \%)$, $\mathrm{T}_{2}(11.19 \%), \mathrm{T}_{1}(11.64 \%)$, at 75DAT lowest recorded was at $\mathrm{T}_{7}(2.94 \%), \mathrm{T}_{6}(2.96 \%)$, $\mathrm{T}_{4}(7.63 \%), \quad \mathrm{T}_{5}(8.81 \%), \quad \mathrm{T}_{3}(8.99 \%)$, $\mathrm{T}_{2}(10.23 \%), \mathrm{T}_{1}(11.21 \%)$, and at 90DAT lowest recorded shoot infestation was in $\mathrm{T}_{7}(1.01 \%)$ followed by $\mathrm{T}_{6}(1.04 \%), \mathrm{T}_{5}(5.19 \%)$, $\mathrm{T}_{4}(5.61 \%), \mathrm{T}_{3}(6.10 \%), \mathrm{T}_{2}(7.76 \%), \mathrm{T}_{1}(8.54 \%)$ against untreated control at $45,60,75,90 \mathrm{DAT}$ was $19.05 \%, 15.18 \%, 11.36 \%, 9.61 \%$ respectively.

Percentage reduction of shoot infestation was maximum in $\mathrm{T}_{7}$ that is $89.49 \%$ followed by $\mathrm{T}_{6}(89.17 \%), \quad \mathrm{T}_{5}(45.99 \%), \quad \mathrm{T}_{4}(41.62 \%)$, $\mathrm{T}_{3}(36.52 \%), \quad \mathrm{T}_{2}(19.25 \%) \quad$ and $\quad \mathrm{T}_{1}(11.13 \%)$ respectively.

Similar trend was recorded on shoot infestation during Kharif of 2016-2017. Shoot infestation before treatment varied from $14.24 \%$ to $20.02 \%$ in different treatments. Shoot infestation percentage at 15DAT ranged lowest at $\mathrm{T}_{7}(17.75 \%)$ followed by $\mathrm{T}_{6}(17.72 \%)$, $\mathrm{T}_{3}(14.24 \%), \quad \mathrm{T}_{4}(16.22 \%), \quad \mathrm{T}_{2}(16.28 \%)$, $\mathrm{T}_{1}(17.13 \%), \quad \mathrm{T}_{5}(18.36 \%)$ against $20.02 \%$ untreated control, at 30DAT lowest $\mathrm{T}_{7}(19.97 \%), \quad \mathrm{T}_{6}(9.98 \%), \quad \mathrm{T}_{4}(12.10 \%)$, $\mathrm{T}_{3}(12.17 \%), \quad \mathrm{T}_{5}(12.67 \%), \quad \mathrm{T}_{2}(13.19 \%) \quad$ and $\mathrm{T}_{1}(14.24 \%)$ against $20.43 \%$ untreated control, at 45DAT lowest infestation $\operatorname{inT}_{7}(5.20 \%)$, $\mathrm{T}_{6}(5.22 \%), \quad \mathrm{T}_{3}(10.08 \%), \quad \mathrm{T}_{4}(10.12 \%)$, $\mathrm{T}_{5}(10.60 \%), \mathrm{T}(11.66 \%), \mathrm{T}_{1}(12.13 \%)$ against untreated control $19.19 \%$, at 60DAT lowest percent of shoot infestation is in $\mathrm{T}_{7}(4.27 \%)$ followed by $\mathrm{T}_{6}(4.28 \%), \quad \mathrm{T}_{5}(9.02 \%)$, $\mathrm{T}_{3}(9.16 \%), \quad \mathrm{T}_{4}(9.23 \%), \quad \mathrm{T}_{2}(11.07 \%)$, $\mathrm{T}_{1}(11.49 \%)$ against untreated control $17.39 \%$, at 75 DAT lowest percent infestation was found in $\mathrm{T}_{7}(2.80 \%)$ followed by $\mathrm{T}_{6}(2.81 \%)$, $\mathrm{T}_{4}(7.83 \%), \quad \mathrm{T}_{3}(8.74 \%), \quad \mathrm{T}_{5}(8.75 \%)$, $\mathrm{T}_{2}(10.12 \%), \quad \mathrm{T}_{1}(10.19 \%)$ against untreated control $14.18 \%$ and at 90DAT percentage of shoot infestation lowest recorded at $\mathrm{T}_{7}(1.17 \%)$ followed by $\mathrm{T}_{6}(1.18 \%), \quad \mathrm{T}_{4}(5.59 \%)$, $\mathrm{T}_{5}(5.62 \%), \quad \mathrm{T}_{3}(6.67 \%), \quad \mathrm{T}_{2}(7.26 \%) \quad$ and $\mathrm{T}_{1}(8.69 \%)$ against $9.73 \%$ (untreated control). Percentage reduction of shoot infestation against the untreated check was highest in $\mathrm{T}_{7}$ with $87.98 \%$ followed by was $\mathrm{T}_{6}(87.87 \%)$, $\mathrm{T}_{5}(62.24 \%), \quad \mathrm{T}_{4}(42.55 \%), \quad \mathrm{T}_{3}(34.53 \%)$, $\mathrm{T}_{2}(25.39 \%)$ and $\mathrm{T}_{1}(10.69 \%)$ respectively.

Studies on the biology of T.chilonis bred on eggs of C.cephalonica and L.orbonalis indicated that all the life stages of T.chilonis liked egg-larval, pupal and adult stages were shorter in duration when bred on eggs of L.orbonalis, this may be due to smaller egg size, have been earlier described by Chowdhury et al., (2016).

The effect of T.chilonis reared on eggs of C.cephalonica and L.orbonalis in controlling shoot and fruit borer in brinjal indicated that, T.chilonis reared on the eggs of L.orbonalis could control the borer more efficiently than the ones whuch were reared on the eggs of C.cephalonica. Release of $50000 \mathrm{each} / \mathrm{ha}$ of T.chilonis reared on eggs of C.cephalonica and L.orbonalis reduced the shoot infestation to the extent of $11.13,41.62 \%$ and 10.69 , $42.55 \%$ respectively in 2016-2017 indicating the superiority of $T$. chilonis bred on the eggs of $L$. orbonalis (Table 1-4) 
Int.J.Curr.Microbiol.App.Sci (2021) 10(02): 1524-1533

Table .1 Comparative effect of T.chilonis bred on eggs of C.cephalonica and L.orbonalis in brinjal shoots during 2015-16

\begin{tabular}{|c|c|c|c|c|c|c|c|c|}
\hline \multirow[b]{2}{*}{ Treatments } & \multicolumn{7}{|c|}{ Shoot infestation (\%) } & \multirow{2}{*}{$\begin{array}{c}\% \\
\text { Reduction } \\
\text { Of shoot } \\
\text { infestation } \\
\text { over control }\end{array}$} \\
\hline & $\begin{array}{c}\text { Pre } \\
\text { treatment }\end{array}$ & 15 DAT & 30 DAT & 45 DAT & 60 DAT & 60 DAT & 90 DAT & \\
\hline $\mathrm{T}_{1}$ & $\begin{array}{l}17.16 \\
(4.14)\end{array}$ & $\begin{array}{l}14.56 \\
(3.82)\end{array}$ & $\begin{array}{l}12.31 \\
(3.51)\end{array}$ & $\begin{array}{l}12.05 \\
(3.47)\end{array}$ & $\begin{array}{l}11.64 \\
(3.41)\end{array}$ & $\begin{array}{l}11.21 \\
(3.35)\end{array}$ & $\begin{array}{c}8.54 \\
(2.92)\end{array}$ & 11.13 \\
\hline $\mathrm{T}_{2}$ & $\begin{array}{l}16.89 \\
(4.29)\end{array}$ & $\begin{array}{l}13.88 \\
(4.68)\end{array}$ & $\begin{array}{c}12.17 \\
(3.48)\end{array}$ & $\begin{array}{l}11.85 \\
(3.44)\end{array}$ & $\begin{array}{l}11.19 \\
(3.35)\end{array}$ & $\begin{array}{c}10.23 \\
(3.20)\end{array}$ & $\begin{array}{c}7.76 \\
(2.79)\end{array}$ & 19.25 \\
\hline $\mathrm{T}_{3}$ & $\begin{array}{l}14.21 \\
(3.76)\end{array}$ & $\begin{array}{l}12.01 \\
(3.46)\end{array}$ & $\begin{array}{l}10.81 \\
(3.29)\end{array}$ & $\begin{array}{c}10.03 \\
(3.17)\end{array}$ & $\begin{array}{c}9.24 \\
(3.04)\end{array}$ & $\begin{array}{c}8.99 \\
(2.99)\end{array}$ & $\begin{array}{c}6.10 \\
(2.47)\end{array}$ & 36.52 \\
\hline $\mathrm{T}_{4}$ & $\begin{array}{l}16.92 \\
(4.03) \\
\end{array}$ & $\begin{array}{l}12.27 \\
(3.50) \\
\end{array}$ & $\begin{array}{l}11.52 \\
(3.39) \\
\end{array}$ & $\begin{array}{l}10.30 \\
(3.21) \\
\end{array}$ & $\begin{array}{c}9.04 \\
(3.01)\end{array}$ & $\begin{array}{c}7.63 \\
(2.76) \\
\end{array}$ & $\begin{array}{c}5.61 \\
(2.37) \\
\end{array}$ & 41.62 \\
\hline $\mathrm{T}_{5}$ & $\begin{array}{l}18.01 \\
(4.24)\end{array}$ & $\begin{array}{l}12.02 \\
(3.46)\end{array}$ & $\begin{array}{l}11.49 \\
(3.39)\end{array}$ & $\begin{array}{l}10.81 \\
(3.29)\end{array}$ & $\begin{array}{c}9.11 \\
(3.02)\end{array}$ & $\begin{array}{c}8.81 \\
(2.97)\end{array}$ & $\begin{array}{c}5.19 \\
(2.28)\end{array}$ & 45.99 \\
\hline $\mathrm{T}_{6}$ & $\begin{array}{l}17.14 \\
(4.14)\end{array}$ & $\begin{array}{c}9.62 \\
(3.11)\end{array}$ & $\begin{array}{c}7.53 \\
(2.74)\end{array}$ & $\begin{array}{c}5.36 \\
(2.38)\end{array}$ & $\begin{array}{c}4.65 \\
(2.16)\end{array}$ & $\begin{array}{c}2.96 \\
(1.72)\end{array}$ & $\begin{array}{c}1.04 \\
(1.01)\end{array}$ & 89.17 \\
\hline $\mathrm{T}_{7}$ & $\begin{array}{l}17.20 \\
(4.15)\end{array}$ & $\begin{array}{c}9.61 \\
(3.11)\end{array}$ & $\begin{array}{c}7.52 \\
(2.74)\end{array}$ & $\begin{array}{c}5.34 \\
(2.31)\end{array}$ & $\begin{array}{c}4.64 \\
(2.15)\end{array}$ & $\begin{array}{c}2.94 \\
(1.71)\end{array}$ & $\begin{array}{c}1.01 \\
(1.00)\end{array}$ & 89.49 \\
\hline $\mathrm{T}_{8}$ & $\begin{array}{l}20.18 \\
(4.49)\end{array}$ & $\begin{array}{l}20.66 \\
(4.54)\end{array}$ & $\begin{array}{l}19.13 \\
(4.37)\end{array}$ & $\begin{array}{l}19.05 \\
(4.36)\end{array}$ & $\begin{array}{l}15.18 \\
(3.89)\end{array}$ & $\begin{array}{l}11.36 \\
(3.38)\end{array}$ & $\begin{array}{l}9.61 \\
(3.1)\end{array}$ & \\
\hline $\mathrm{SE}(\mathrm{m})) \pm$ & 0.24 & 0.47 & 0.54 & 0.67 & 0.57 & 0.55 & 0.69 & \\
\hline $\mathrm{CD}(\mathrm{p}=0.05)$ & 0.73 & 1.42 & 1.63 & 2.03 & 1.73 & 1.67 & 2.10 & \\
\hline
\end{tabular}

Figures in parentheses are $\sqrt{ }$ values. 
Table.2 Comparative effect of T.chilonis bred on eggs of C.cephalonica and L.orbonalis in brinjal shoots during 2016-17

\begin{tabular}{|c|c|c|c|c|c|c|c|c|}
\hline \multirow[b]{2}{*}{ Treatments } & \multicolumn{7}{|c|}{ Shoot infestation (\%) } & \multirow{2}{*}{$\begin{array}{c}\% \\
\text { Reduction } \\
\text { of shoot } \\
\text { infestation } \\
\text { over control }\end{array}$} \\
\hline & $\begin{array}{c}\text { Pre } \\
\text { treatment }\end{array}$ & 15 DAT & 30 DAT & 45 DAT & 60 DAT & 60 DAT & 90 DAT & \\
\hline $\mathrm{T}_{1}$ & $\begin{array}{l}17.73 \\
(4.21)\end{array}$ & $\begin{array}{l}14.24 \\
(3.77)\end{array}$ & $\begin{array}{l}12.39 \\
(3.52)\end{array}$ & $\begin{array}{l}12.13 \\
(3.48)\end{array}$ & $\begin{array}{l}11.49 \\
(3.39)\end{array}$ & $\begin{array}{l}10.19 \\
(3.19)\end{array}$ & $\begin{array}{c}8.69 \\
(2.95)\end{array}$ & 10.69 \\
\hline $\mathrm{T}_{2}$ & $\begin{array}{l}16.28 \\
(4.03)\end{array}$ & $\begin{array}{l}13.19 \\
(3.63)\end{array}$ & $\begin{array}{l}12.13 \\
(3.48)\end{array}$ & $\begin{array}{l}11.66 \\
(3.41)\end{array}$ & $\begin{array}{l}11.07 \\
(3.33)\end{array}$ & $\begin{array}{l}10.12 \\
(3.18)\end{array}$ & $\begin{array}{c}7.26 \\
(2.69)\end{array}$ & 25.39 \\
\hline $\mathrm{T}_{3}$ & $\begin{array}{l}14.24 \\
(3.77)\end{array}$ & $\begin{array}{l}12.17 \\
(3.49)\end{array}$ & $\begin{array}{l}10.38 \\
(3.22)\end{array}$ & $\begin{array}{l}10.08 \\
(3.17)\end{array}$ & $\begin{array}{c}9.16 \\
(3.03)\end{array}$ & $\begin{array}{c}8.74 \\
(2.96)\end{array}$ & $\begin{array}{c}6.37 \\
(2.52)\end{array}$ & 34.53 \\
\hline $\mathrm{T}_{4}$ & $\begin{array}{l}16.22 \\
(4.03)\end{array}$ & $\begin{array}{l}12.10 \\
(3.48)\end{array}$ & $\begin{array}{l}11.66 \\
(3.41)\end{array}$ & $\begin{array}{l}10.12 \\
(3.18)\end{array}$ & $\begin{array}{c}9.23 \\
(3.04)\end{array}$ & $\begin{array}{c}7.83 \\
(2.80)\end{array}$ & $\begin{array}{c}5.59 \\
(2.36)\end{array}$ & 42.55 \\
\hline $\mathrm{T}_{5}$ & $\begin{array}{l}18.36 \\
(4.28)\end{array}$ & $\begin{array}{l}12.67 \\
(3.56)\end{array}$ & $\begin{array}{l}11.13 \\
(3.37)\end{array}$ & $\begin{array}{l}10.60 \\
(3.26)\end{array}$ & $\begin{array}{c}9.02 \\
(3.00)\end{array}$ & $\begin{array}{c}8.75 \\
(2.99)\end{array}$ & $\begin{array}{c}5.62 \\
(2.37)\end{array}$ & 62.24 \\
\hline $\mathrm{T}_{6}$ & $\begin{array}{l}17.72 \\
(4.21)\end{array}$ & $\begin{array}{c}9.98 \\
(3.16)\end{array}$ & $\begin{array}{c}7.10 \\
(2.66)\end{array}$ & $\begin{array}{c}5.22 \\
(2.28)\end{array}$ & $\begin{array}{c}4.28 \\
(2.07)\end{array}$ & $\begin{array}{c}2.81 \\
(1.68)\end{array}$ & $\begin{array}{c}1.18 \\
(1.09)\end{array}$ & 87.87 \\
\hline $\mathrm{T}_{7}$ & $\begin{array}{l}17.75 \\
(4.21)\end{array}$ & $\begin{array}{c}9.97 \\
(3.16)\end{array}$ & $\begin{array}{l}7.08 \\
(2.66)\end{array}$ & $\begin{array}{c}5.20 \\
(2.28)\end{array}$ & $\begin{array}{c}4.27 \\
(2.07)\end{array}$ & $\begin{array}{c}2.80 \\
(1.67)\end{array}$ & $\begin{array}{c}1.17 \\
(1.08)\end{array}$ & 87.98 \\
\hline $\mathrm{T}_{8}$ & $\begin{array}{l}20.02 \\
(4.47)\end{array}$ & $\begin{array}{l}20.43 \\
(4.52)\end{array}$ & $\begin{array}{l}19.40 \\
(4.40)\end{array}$ & $\begin{array}{l}19.19 \\
(4.38)\end{array}$ & $\begin{array}{l}17.35 \\
(4.16)\end{array}$ & $\begin{array}{l}14.18 \\
(3.77)\end{array}$ & $\begin{array}{c}9.73 \\
(3.12)\end{array}$ & \\
\hline $\begin{array}{c}\mathrm{SE}(\mathrm{m})) \pm \\
\mathrm{CD}(\mathrm{p}=0.05)\end{array}$ & $\begin{array}{l}0.15 \\
045\end{array}$ & $\begin{array}{l}0.45 \\
1.36\end{array}$ & $\begin{array}{l}0.57 \\
1.72\end{array}$ & $\begin{array}{l}0.69 \\
2.10\end{array}$ & $\begin{array}{l}1.95 \\
2.90\end{array}$ & $\begin{array}{l}0.69 \\
2.10\end{array}$ & $\begin{array}{l}0.67 \\
2.03\end{array}$ & \\
\hline
\end{tabular}

Figures in parentheses are $\sqrt{ }$ values. 
Table.3 Comparative effect of T.chilonis bred on eggs of C.cephalonica and L.orbonalis in brinjal fruits during 2015-16

\begin{tabular}{|c|c|c|c|c|c|c|c|c|c|c|c|c|}
\hline \multicolumn{12}{|c|}{ Fruit infestation (\%) } & \multirow[b]{2}{*}{$\begin{array}{c}\% \\
\text { Reduction of } \\
\text { shoot } \\
\text { infestation } \\
\text { over control }\end{array}$} \\
\hline $\begin{array}{c}\text { Treatme } \\
\text { nts }\end{array}$ & $\begin{array}{c}\text { Pre } \\
\text { treatme } \\
\text { nt }\end{array}$ & $\begin{array}{c}\mathbf{5 0} \\
\text { DAT }\end{array}$ & $\begin{array}{c}60 \\
\text { DAT }\end{array}$ & $\begin{array}{c}70 \\
\text { DAT }\end{array}$ & $\begin{array}{c}80 \\
\text { DAT }\end{array}$ & $\begin{array}{c}90 \\
\text { DAT }\end{array}$ & $\begin{array}{c}100 \\
\text { DAT }\end{array}$ & $\begin{array}{c}110 \\
\text { DAT }\end{array}$ & $\begin{array}{c}120 \\
\text { DAT }\end{array}$ & $\begin{array}{c}130 \\
\text { DAT }\end{array}$ & $\begin{array}{c}140 \\
\text { DAT }\end{array}$ & \\
\hline $\mathrm{T}_{1}$ & $\begin{array}{c}35.85 \\
(36.81)\end{array}$ & $\begin{array}{c}33.60 \\
(35.43)\end{array}$ & $\begin{array}{c}33.19 \\
(35.18)\end{array}$ & $\begin{array}{c}31.44 \\
(34.10)\end{array}$ & $\begin{array}{c}30.20 \\
(33.34)\end{array}$ & $\begin{array}{c}28.04 \\
(31.95)\end{array}$ & $\begin{array}{c}26.86 \\
(31.24)\end{array}$ & $\begin{array}{c}26.19 \\
(30.79)\end{array}$ & $\begin{array}{c}24.78 \\
(29.87)\end{array}$ & $\begin{array}{c}21.81 \\
(27.83) \\
\end{array}$ & $\begin{array}{c}19.27 \\
(26.06)\end{array}$ & $\begin{array}{c}73.11 \\
(58.76) \\
\end{array}$ \\
\hline $\mathrm{T}_{2}$ & $\begin{array}{c}34.79 \\
(36.15) \\
\end{array}$ & $\begin{array}{c}32.26 \\
(34.63)\end{array}$ & $\begin{array}{c}30.12 \\
(33.27)\end{array}$ & $\begin{array}{c}27.56 \\
(31.69)\end{array}$ & $\begin{array}{c}27.19 \\
(31.44)\end{array}$ & $\begin{array}{c}25.40 \\
(30.26)\end{array}$ & $\begin{array}{c}22.22 \\
(28.11)\end{array}$ & $\begin{array}{c}22.03 \\
(27.97)\end{array}$ & $\begin{array}{c}20.85 \\
(27.13)\end{array}$ & $\begin{array}{c}20.66 \\
(27.06)\end{array}$ & $\begin{array}{c}18.73 \\
(25.62)\end{array}$ & $\begin{array}{c}73.86 \\
(59.28) \\
\end{array}$ \\
\hline $\mathrm{T}_{3}$ & $\begin{array}{c}36.11 \\
(36.33)\end{array}$ & $\begin{array}{c}32.01 \\
(34.45)\end{array}$ & $\begin{array}{c}30.57 \\
(33.58)\end{array}$ & $\begin{array}{c}26.86 \\
(31.24)\end{array}$ & $\begin{array}{c}23.74 \\
(29.13)\end{array}$ & $\begin{array}{c}23.33 \\
(28.86)\end{array}$ & $\begin{array}{c}21.81 \\
(27.83)\end{array}$ & $\begin{array}{c}19.70 \\
(26.35)\end{array}$ & $\begin{array}{c}19.12 \\
(25.92)\end{array}$ & $\begin{array}{c}19.36 \\
(26.13)\end{array}$ & $\begin{array}{c}17.50 \\
(24.73)\end{array}$ & $\begin{array}{c}75.58 \\
(60.40)\end{array}$ \\
\hline $\mathrm{T}_{4}$ & $\begin{array}{c}34.80 \\
(36.15)\end{array}$ & $\begin{array}{c}31.16 \\
(33.95)\end{array}$ & $\begin{array}{c}29.82 \\
(33.09)\end{array}$ & $\begin{array}{c}29.13 \\
(32.65)\end{array}$ & $\begin{array}{c}27.66 \\
(31.69)\end{array}$ & $\begin{array}{c}24.17 \\
(29.47)\end{array}$ & $\begin{array}{c}24.01 \\
(29.33)\end{array}$ & $\begin{array}{c}22.67 \\
(28.45)\end{array}$ & $\begin{array}{c}22.03 \\
(27.97)\end{array}$ & $\begin{array}{c}18.73 \\
(25.62)\end{array}$ & $\begin{array}{c}16.06 \\
(23.66)\end{array}$ & $\begin{array}{c}77.64 \\
(61.75)\end{array}$ \\
\hline $\mathrm{T}_{5}$ & $\begin{array}{c}35.23 \\
(36.39)\end{array}$ & $\begin{array}{c}30.08 \\
(33.71)\end{array}$ & $\begin{array}{c}27.66 \\
(31.75) \\
\end{array}$ & $\begin{array}{c}24.19 \\
(29.47)\end{array}$ & $\begin{array}{c}24.01 \\
(29.33)\end{array}$ & $\begin{array}{c}21.78 \\
(27.83)\end{array}$ & $\begin{array}{c}21.40 \\
(27.56)\end{array}$ & $\begin{array}{c}18.62 \\
(25.55)\end{array}$ & $\begin{array}{c}15.41 \\
(23.11)\end{array}$ & $\begin{array}{c}16.40 \\
(23.89)\end{array}$ & $\begin{array}{c}14.98 \\
(22.71)\end{array}$ & $\begin{array}{l}79.10 \\
(62.80)\end{array}$ \\
\hline $\mathrm{T}_{6}$ & $\begin{array}{c}37.50 \\
(37.76)\end{array}$ & $\begin{array}{c}28.44 \\
(32.20)\end{array}$ & $\begin{array}{c}26.20 \\
(30.79)\end{array}$ & $\begin{array}{c}22.85 \\
(28.38)\end{array}$ & $\begin{array}{c}19.77 \\
(26.35)\end{array}$ & $\begin{array}{c}19.58 \\
(26.26)\end{array}$ & $\begin{array}{c}16.68 \\
(24.12)\end{array}$ & $\begin{array}{c}16.33 \\
(23.81)\end{array}$ & $\begin{array}{c}15.11 \\
(22.87)\end{array}$ & $\begin{array}{c}12.72 \\
(20.88)\end{array}$ & $\begin{array}{c}9.67 \\
(18.15)\end{array}$ & $\begin{array}{c}86.51 \\
(68.44)\end{array}$ \\
\hline $\mathrm{T}_{7}$ & $\begin{array}{c}37.52 \\
(37.76)\end{array}$ & $\begin{array}{c}28.42 \\
(32.20)\end{array}$ & $\begin{array}{c}26.19 \\
(30.79)\end{array}$ & $\begin{array}{c}22.83 \\
(28.52)\end{array}$ & $\begin{array}{c}19.76 \\
(26.35)\end{array}$ & $\begin{array}{c}19.56 \\
(26.21)\end{array}$ & $\begin{array}{c}16.65 \\
(24.04)\end{array}$ & $\begin{array}{c}16.31 \\
(23.81)\end{array}$ & $\begin{array}{c}15.10 \\
(22.87)\end{array}$ & $\begin{array}{c}12.70 \\
(20.88)\end{array}$ & $\begin{array}{c}9.66 \\
(18.12)\end{array}$ & $\begin{array}{c}86.52 \\
(68.44)\end{array}$ \\
\hline $\mathrm{T}_{8}$ & $\begin{array}{c}40.19 \\
(39.35)\end{array}$ & $\begin{array}{c}43.10 \\
(41.03)\end{array}$ & $\begin{array}{c}47.03 \\
(43.28)\end{array}$ & $\begin{array}{c}51.39 \\
(45.80)\end{array}$ & $\begin{array}{c}55.16 \\
(47.98)\end{array}$ & $\begin{array}{c}60.46 \\
(51.06)\end{array}$ & $\begin{array}{c}64.12 \\
(53.19)\end{array}$ & $\begin{array}{c}67.00 \\
(54.94)\end{array}$ & $\begin{array}{c}69.16 \\
(56.29)\end{array}$ & $\begin{array}{c}70.31 \\
(56.98)\end{array}$ & $\begin{array}{c}71.66 \\
(57.80)\end{array}$ & \\
\hline $\mathrm{SE}(\mathrm{m}) \pm$ & $(0.48)$ & $(0.60)$ & $(0.36)$ & $(0.43)$ & $(0.92)$ & $(0.48)$ & $(0.85)$ & $(0.77)$ & $(0.70)$ & $(0.70)$ & $(0.53)$ & \\
\hline $\begin{array}{c}\mathrm{CD}(\mathrm{p}=0 \\
05)\end{array}$ & (1.45) & (1.82) & (1.10) & (1.30) & $(2.78)$ & (1.45) & (2.58) & (2.33) & $(2.12)$ & $(2.12)$ & (1.61) & \\
\hline
\end{tabular}

Figures in parentheses are $\sqrt{ }$ values. 
Table.4 Comparative effect of T.chilonis bred on eggs of C.cephalonica and L.orbonalis in brinjal fruits during 2016-17

\begin{tabular}{|c|c|c|c|c|c|c|c|c|c|c|c|c|}
\hline \multirow[b]{2}{*}{ Treatments } & \multicolumn{11}{|c|}{ Fruit infestation (\%) } & \multirow{2}{*}{$\begin{array}{c}\% \\
\text { Reduction } \\
\text { Of shoot } \\
\text { Infestation } \\
\text { Over control }\end{array}$} \\
\hline & $\begin{array}{c}\text { Pre } \\
\text { treatment }\end{array}$ & $\begin{array}{c}50 \\
\text { DAT }\end{array}$ & $\begin{array}{c}60 \\
\text { DAT }\end{array}$ & $\begin{array}{c}70 \\
\text { DAT }\end{array}$ & $\begin{array}{c}80 \\
\text { DAT }\end{array}$ & $\begin{array}{c}90 \\
\text { DAT }\end{array}$ & $\begin{array}{c}100 \\
\text { DAT }\end{array}$ & $\begin{array}{c}110 \\
\text { DAT }\end{array}$ & $\begin{array}{c}120 \\
\text { DAT }\end{array}$ & $\begin{array}{c}130 \\
\text { DAT }\end{array}$ & $\begin{array}{c}140 \\
\text { DAT }\end{array}$ & \\
\hline $\mathrm{T}_{1}$ & $\begin{array}{c}35.60 \\
(36.63)\end{array}$ & $\begin{array}{c}33.24 \\
(35.18)\end{array}$ & $\begin{array}{c}33.02 \\
(35.06)\end{array}$ & $\begin{array}{c}31.14 \\
(33.83)\end{array}$ & $\begin{array}{c}30.12 \\
(33.27)\end{array}$ & $\begin{array}{c}28.09 \\
(32.01)\end{array}$ & $\begin{array}{c}26.73 \\
(31.11) \\
\end{array}$ & $\begin{array}{c}26.12 \\
(30.72)\end{array}$ & $\begin{array}{c}25.01 \\
(30.00)\end{array}$ & $\begin{array}{c}22.08 \\
(28.04)\end{array}$ & $\begin{array}{c}21.30 \\
(27.49)\end{array}$ & $\begin{array}{c}69.24 \\
(56.29)\end{array}$ \\
\hline $\mathrm{T}_{2}$ & $\begin{array}{c}34.56 \\
(36.03)\end{array}$ & $\begin{array}{c}32.35 \\
(34.70)\end{array}$ & $\begin{array}{c}30.41 \\
(33.46)\end{array}$ & $\begin{array}{c}27.39 \\
(31.55)\end{array}$ & $\begin{array}{c}27.04 \\
(31.31)\end{array}$ & $\begin{array}{c}25.17 \\
(30.13)\end{array}$ & $\begin{array}{c}22.42 \\
(28.25)\end{array}$ & $\begin{array}{c}22.16 \\
(28.11)\end{array}$ & $\begin{array}{c}20.43 \\
(26.85)\end{array}$ & $\begin{array}{c}20.05 \\
(26.56)\end{array}$ & $\begin{array}{c}17.14 \\
(24.43)\end{array}$ & $\begin{array}{c}75.26 \\
(60.20)\end{array}$ \\
\hline $\mathrm{T}_{3}$ & $\begin{array}{c}36.13 \\
(36.93)\end{array}$ & $\begin{array}{c}32.12 \\
(34.51)\end{array}$ & $\begin{array}{c}30.42 \\
(33.46)\end{array}$ & $\begin{array}{c}26.03 \\
(30.66)\end{array}$ & $\begin{array}{c}23.52 \\
(29.00)\end{array}$ & $\begin{array}{c}23.81 \\
(29.20)\end{array}$ & $\begin{array}{c}21.88 \\
(27.90)\end{array}$ & $\begin{array}{c}19.59 \\
(26.28)\end{array}$ & $\begin{array}{c}19.14 \\
(25.92)\end{array}$ & $\begin{array}{c}17.21 \\
(24.50)\end{array}$ & $\begin{array}{c}15.46 \\
(23.19)\end{array}$ & $\begin{array}{c}77.68 \\
(61.82)\end{array}$ \\
\hline $\mathrm{T}_{4}$ & $\begin{array}{c}34.43 \\
(35.91)\end{array}$ & $\begin{array}{c}31.22 \\
(33.96)\end{array}$ & $\begin{array}{c}29.16 \\
(32.71)\end{array}$ & $\begin{array}{c}29.46 \\
(32.90)\end{array}$ & $\begin{array}{c}27.01 \\
(31.31)\end{array}$ & $\begin{array}{c}24.63 \\
(29.73)\end{array}$ & $\begin{array}{c}24.02 \\
(29.33)\end{array}$ & $\begin{array}{c}22.81 \\
(28.52)\end{array}$ & $\begin{array}{c}22.23 \\
(28.11)\end{array}$ & $\begin{array}{c}18.33 \\
(25.33)\end{array}$ & $\begin{array}{c}15.44 \\
(23.11)\end{array}$ & $\begin{array}{c}77.71 \\
(61.82)\end{array}$ \\
\hline $\mathrm{T}_{5}$ & $\begin{array}{c}35.12 \\
(36.33)\end{array}$ & $\begin{array}{c}30.98 \\
(32.27)\end{array}$ & $\begin{array}{c}27.05 \\
(31.31)\end{array}$ & $\begin{array}{c}24.89 \\
(29.93)\end{array}$ & $\begin{array}{c}24.01 \\
(29.33)\end{array}$ & $\begin{array}{c}21.87 \\
(27.90)\end{array}$ & $\begin{array}{c}21.36 \\
(27.56)\end{array}$ & $\begin{array}{c}18.52 \\
(25.48)\end{array}$ & $\begin{array}{c}15.56 \\
(23.26)\end{array}$ & $\begin{array}{c}15.30 \\
(23.03)\end{array}$ & $\begin{array}{c}12.10 \\
(20.36)\end{array}$ & $\begin{array}{c}82.53 \\
(65.27)\end{array}$ \\
\hline $\mathrm{T}_{6}$ & $\begin{array}{c}37.15 \\
(37.58)\end{array}$ & $\begin{array}{c}28.46 \\
(32.20)\end{array}$ & $\begin{array}{c}26.17 \\
(30.79)\end{array}$ & $\begin{array}{c}22.77 \\
(28.52)\end{array}$ & $\begin{array}{c}19.46 \\
(26.21)\end{array}$ & $\begin{array}{c}19.68 \\
(26.35)\end{array}$ & $\begin{array}{c}15.84 \\
(23.42)\end{array}$ & $\begin{array}{c}15.37 \\
(23.11)\end{array}$ & $\begin{array}{c}15.07 \\
(22.79)\end{array}$ & $\begin{array}{c}12.64 \\
(20.79)\end{array}$ & $\begin{array}{c}9.67 \\
(18.12)\end{array}$ & $\begin{array}{c}86.04 \\
(68.03)\end{array}$ \\
\hline $\mathrm{T}_{7}$ & $\begin{array}{c}37.17 \\
(37.58)\end{array}$ & $\begin{array}{c}28.43 \\
(32.20)\end{array}$ & $\begin{array}{c}26.15 \\
(30.79)\end{array}$ & $\begin{array}{c}22.76 \\
(28.52)\end{array}$ & $\begin{array}{c}19.44 \\
(26.13)\end{array}$ & $\begin{array}{c}19.65 \\
(26.28)\end{array}$ & $\begin{array}{c}15.83 \\
(23.42)\end{array}$ & $\begin{array}{c}15.35 \\
(23.03)\end{array}$ & $\begin{array}{c}15.06 \\
(22.79)\end{array}$ & $\begin{array}{c}12.62 \\
(20.79)\end{array}$ & $\begin{array}{c}9.66 \\
(18.02)\end{array}$ & $\begin{array}{c}86.05 \\
(68.11)\end{array}$ \\
\hline $\mathrm{T}_{8}$ & $\begin{array}{c}39.78 \\
(39.11)\end{array}$ & $\begin{array}{c}42.13 \\
(40.46)\end{array}$ & $\begin{array}{c}46.23 \\
(42.82)\end{array}$ & $\begin{array}{c}50.31 \\
(45.17)\end{array}$ & $\begin{array}{c}54.23 \\
(47.41)\end{array}$ & $\begin{array}{c}59.61 \\
(50.53)\end{array}$ & $\begin{array}{c}63.89 \\
(53.07)\end{array}$ & $\begin{array}{c}66.19 \\
(54.45)\end{array}$ & $\begin{array}{c}68.53 \\
(55.86) \\
\end{array}$ & $\begin{array}{c}69.62 \\
(56.54)\end{array}$ & $\begin{array}{c}69.26 \\
(56.35)\end{array}$ & \\
\hline $\mathrm{SE}(\mathrm{m})) \pm$ & 0.36 & 0.85 & 0.66 & 1.04 & 0.73 & 0.85 & 0.72 & 0.73 & 0.92 & 0.73 & 0.36 & \\
\hline $\mathrm{CD}(\mathrm{p}=0.05)$ & 1.10 & 2.58 & 2.00 & 3.15 & 2.21 & 2.58 & 2.18 & 2.21 & 2.78 & 2.21 & 1.10 & \\
\hline
\end{tabular}

Figures in parentheses are $\sqrt{ }$ values. 
Similarly at $75000 /$ ha rate of release, the reduction was $19.25,45.99 \%$ and $25.39,62.24 \%$ in 2012-13 and 2013-14 respectively, again proving the supremacy of L. orbonalis bred T. chilonis. Similar trend was also found when 100000 parasitoids were released /ha and the reduction in 2012-13 and 2013-14 were $36.52,89.17 \%$ and 34.53 , $87.87 \%$ respectively. This result completely demonstrated that $T$. chilonis bred on the eggs of L. orbonalis is significantly superior in respect of control of the brinjal shoot and fruit borer over T.chilonis bred on the eggs of C.cephalonica. The result of fruit infestation also confirmed superiority T.chilonis bred on the eggs of L.orbonalis by reducing the fruit infestation to the tune of $77.64,79.10$ and $86.51 \%$ in $2012-13$ and $77.71,82.53$ and $86.04 \%$ in $2013-14$ as against $73.11,73.86$ and $75.58 \%$ in 2012-13 and 69.24, 75.26 and $77.71 \%$ in $2013-14$ when the parasitoid was bred on eggs of C.cephalonica. Our observation agrees with the study conducted by Ranjith et al., (2018), This may be due to the continuous breading of the parasitoid for over 30 generations in the eggs of L.orbonalis. Previously attempts have been made successfully by Jalali et al., (2006), Jalali et al., (2016) for production of temperature tolerant Trichogramma by subjecting the parasitoid continuously to high temperature and high insecticide concentrations gradually. Similar attempts have been made here to produce a strain of Trichogramma chilonis exclusively adopted to the brinjal shoot and fruit borer.

\section{Acknowledgement}

The authors gratefully acknowledge the help rendered by the Head of the Department, Department of Entomology, College of Agriculture, OUAT, Bhubaneswar for providing necessary laboratory and field facilities for conducting the experiments.

\section{References}

Alam SN,Rashid MA, Rouf FMA, Jhala RC, Patel JR, Satpathy S. Shivalingaswamy, Rai TM, Wahunderiya SI, Cork A, Ammaranan C, Talekar NS.2003. Development of an integrated pest management strategy for egg plant fruit and shoot borer in South Asia,. Final Technical Report of a DFID funded Project R7465@. AVRDC, Taiwan.

Ghosh SK, Senapati SK. 2009. Seasonal Fluctuation in the Population of Leucinodes orbonalis (Guenée) in the sub-himalayan Region of West Bengal, India and its Control on Eggplant (Solanum melongena L.), Precision Agriculture, 10(5): 443-449.

Jalali S K, Venkatesan T, Murthy K S, Rabindra R J, Lalitha Y. 2006. Vacuum packaging of Corcyra cephalonica (Staiton) eggs to enhance shelf life for parasitization by the egg parasitoid Trichogramma chilonis. Biol. Control,41: 64-67.

Jalali S K, T Venkatesan, K S, Murthy, R Ojha. 2016. Management of Helicoverpa armigera (Hubner) on tomato using insecticide resistance egg parasitoid, Trichogramma chilonis Ishii in farmers' field. Indian Journal of Horticulture, 73: 611-614.

Mainali RP. 2014. Biology and management of eggplant fruit and shoot borer Leucinodes orbonalis (Guenée) (Lepidoptera: Pyralidae), Int J Appl Sci Biotechnology, 2(1): 18-28.

Mishra HP.2008.New promising insecticides for the management of brinjal shoot and fruit borer, Leucinodes orbonalis (Guenée), Pest Management in Horticultural Ecosystems, 14(2): 140147.

Payal Devi, Sahu TK, Bihariahirwar R, Kostha VK. 2014. Field evaluation of 
insecticides for management of shoot and fruit borer, Leucinodes orbonalis (Guenée) in brinjal, The Ecoscan, 6: 463-466.

Prabhat K, Johnsen S. 2000. Life Cycle studies on fruit and shoot borer Leucinodes ornonalis and natural enemies of insect pests of eggplant (Solanum melonena), Journal of Applied Biology, 10(2): 178-184.

Ranjith M, Husain D, Ul Islam S, Ali H, Islam W, Hussain M, Wang F, Wang L. 2018. Effectiveness of Trichogramma chilonis Ishii against spiny bollworm in Okra and susceptibility to insecticides. $J$
Entomol Zool Stud 6:1576-1581.

Regupathy A, NJ Armes, G Asoken, DR Jadhav, RD Soundarajan, DA Russell. 1997. Best method for insecticide resistance management of Helicoverpa armigera. In: International Conference on Integrated Approach to Combating Resistance. A.L. Devonshine (ed.), April 14-16, 1997. IACR, Rothamsted, Harpendle, UK. 116p.

Srinivasan G, Babu PCS. 2008. Sex pheromones for brinjal shoot and fruit borer, Leucinodes orbonalis (Guenée), Indian Journal of Entomology, 62(2): 225-226.

\section{How to cite this article:}

Mou Biswas, Ipsita Mishra and Subhalaxmi Roy. 2021. Comparative Efficacy of Trichogramma chilonis Bred on Corcyra cephalonica and Leucinodes orbonalis (Guenee) Eggs in controlling Shoot and Fruit Borer in Brinjal. Int.J.Curr.Microbiol.App.Sci. 10(02): 1524-1533. doi: https://doi.org/10.20546/ijcmas.2021.1002.182 